\title{
The CyberSport Nexus
}

\author{
By Professor Andy Miah
}

\section{Citation:}

Miah, A. (2014) The CyberSport Nexus, in Billings, A.\& Hardin, M. The Routledge Handbook of Sport and New Media, Oxon \& New York, Routledge, pp.76-86.

"Exercise machines increasingly incorporate computer-controlled motion and force feedback and will eventually become reactive robotic sports partners....Today's rudimentary, narrowband video games will evolve into physically engaging telesports" (Mitchell 1995, p. 19).

Mitchell's vision of sport's future was one of the first to foreground the integration of digital systems within a broader context of technocultural change. His articulation of how sports would be played within cities of bits reveals the intimate connection between organized, competitive sports and the urban environment, where digital innovation is embedded within the build environment. Yet, Mitchell's attention did not attend to the wider context of the digital future he foresaw, where other aspects of the sport experience would be transformed by digital technology and where the convergence of physical and virtual worlds reveals the radical challenge from sport's new media.

As digital environments and sports cultures develop, humanity comes closer to an era of digitally constituted sports experiences, where the primary medium of participation need no longer be a physical playing field or arena, but is a virtual space. While it may seem a long way off to a world where sports take place entirely within digital worlds, this chapter considers how far toward this era sports have come and what may come next. A number of questions arise from this prospect. How are such conditions changing sports experiences, physical activity, and people's experience of their own embodiment? How does digital space change the meaning attributed to sports, their function, and the way in which they give rise to communities of participation? Alternatively, digital innovation invites us to consider whether sports will occupy a different place within our social and cultural lives. Furthermore, one may ask about the consequences of making corporeality 
a surrogate to a virtual presence, which subsequently may create a physical culture that is defined largely by digital interactions. What distinguishes answering these questions today from when Mitchell imagined the future of sports is that we know and can observe some of the initiatives that will shape these emerging realities.

The future is not quite how it looked back then, but this chapter reveals some of the striking resemblances between these periods and the implications presented by the last two decades of digital innovation around sport. In so doing, it describes the CyberSport Nexus as the interface of digital technologies with sport, from virtual realities to social media, arguing that this comprehensive consideration of the new media within sport most adequately explains what is at stake within this transformation. It aims to understand the implications of sports becoming digital enterprises. Experiences within virtual worlds have already become inextricable from many other forms of social and

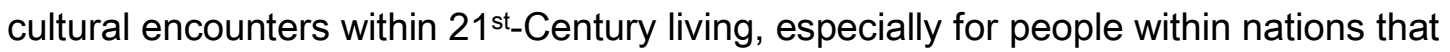
have a digital infrastructure. From remotely conducted surgical procedures in medicine to the global economy, life online is a constitutive element of many societies and, in varying fashions, participation cuts across other technological divides. Yet, life online remains the subject of popular controversy, with continuing allegations of computer game playing leading to violent, anti-social behavior (Anderson, Gentile \& Buckley, 2007; Comstock \& Scharrer, 2007), or screen time promoting sedentary lifestyles or more risky childhoods (Byron, 2008). These allegations are often advanced against specific, prominent examples of digital experiences - such as computer games that depict criminal or anti-social acts - but they contrast with other studies of digital culture, which reveal the capacity of computer games to inspire creativity, physical activity (Marshall et al., 2004), and engagement with the world around us (Ott \& Pozzi, 2011).

Furthermore, there is considerable mistrust in the idea that a more digitally mediated future lends itself to a better world, a more just society, or richer personal relationships. In part, this is why the debate about new media and sport is critically connected to debates about ethics, morality, and the good life. At a time when the onslaught of digital technology appears to be all consuming, it is useful to consider claims about what might be won or lost in the process. To scrutinize these competing claims, it is necessary to locate them within a context of digital participation. Whereas media consumption 20 years ago took place in quite isolated, discrete physical spaces, such as public theatres 
and home living rooms, today's media experiences operate across social spaces, from aircraft to trains, from mountains to swimming pools. Content is delivered across platforms, making it possible for someone to listen to a waterproofed personal music device while swimming and use the same device on a home music system. These possibilities change the debate about the social consequences of media consumption, since the habitus of media exposure is disrupted. Media participation may take place pervasively across an individual's life, creating multi-layered social experiences where previously separate activities become more intimately connected, and where there is blurring of physical space. A person may be watching a film in a cinema while playing a game on a mobile device and sharing what is happening with friends via instant messaging.

In some cases, the social commentary around the benefit of such innovation is made moot by widespread adoption, which marks a significant change in consumer habits. For instance, while e-book readers may present challenges for the book industry, the Amazon Kindle has transformed how people interface with books. Even if there is some compromise to the book's value, e-books provide convenient solutions that appeal because of their adaptability. However, in other cases, there is concern over the potential loss these new media experiences may bring, and it is these considerations that focus our attention in the context of cybersport. For instance, does our evaluation of the worth of digital technology change, if physically demanding elements of game playing are introduced? Alternatively, do we regard new media differently if it can be shown to have a bigger impact on promoting certain values that we care about, such as greater accessibility, technological equality, or participation?

This chapter provides a more extensive insight into how the cybersport nexus will operate by examining some of the key trends. It considers the ways media industries operate through digital technology around sport while also discussing how media technology changes the parameters of what it is to be a participant within the sports world - whether as an athlete, spectator or official. In so doing, it also considers the ethical and moral dimensions of this landscape, discussing how these transformations challenge our understanding of sports, what sports may become, and what value cybersports may have. This chapter also exposes the creative use of emerging digital technology within sports culture in order to reveal the ways in which such practices are 
undergoing significant revision as a result of new technological changes, but also how the culture of physicality that surrounds digital experiences is transforming how we make sense of these developments as either dystopian or utopian. More precisely, I discuss how the advanced use of digital technologies in sports is transforming these practices into new cultural experiences, which are defined by different values and expectations, along with new populations of practitioners.

To further focus the chapter, I examine three dimensions of the sport experience: athletes, spectators, and officials. In each case, I consider what are some of the new digital innovations that are changing the landscape of new media within their field, the key social, moral and ethical challenges this provokes for the world of sport, and what outcomes may arise from such innovation in the long term.

\section{THE ATHLETE}

For as long as organized sports have existed, new media technology has been utilized by athletes, either for training, performance data capture, or through the technology used within the playing field. In a pre-digital era, timing technology, video capture, and simulation devices were among the many examples that describe the sport's world as a space of creative technological innovation, focused on simulation and measurement. Today, athletes benefit from shoes or even prosthetic devices that are created using foot-scanning technology to ensure their equipment perfectly accommodates their unique physical form. Alternatively, athletes have trained within virtual reality environments, as was true of the US Bob Sled team in Nagano 1998, who practiced their sled run in a simulator (Huffman and Hubbard 1996, Levy \& Katz 2007). Simulators have now been used in countless sports, from sailing (Walls et al, 1998) to handball (Bideau et al. 2004) to swimming. For instance, before the London 2012 Olympic Games, computer scientists built a computer game called 'Open Water Warfare' to help swimmers navigate during competition. The game simulates the official Olympic open water venue to help swimmers train, which was not possible to access before the Games.

Today, athletes experience their sport within a data-rich environment, which provides insights into their development and performance, mostly for their expert advisers. In this sense, the knowledge systems that surround athletes are highly dependent on new 
media, which is an ongoing asset to improve performance and our understanding of it. These apparatuses are utilized within established sports practices. Sometimes, they may yield the creation of new sports. Already, a new generation of elite athletes is emerging through the use of new media. Cyberathletes who compete within computer game tournaments undertake activities that are often highly demanding physically and, perhaps more importantly, are co-produced by media broadcasters in ways remarkably similar to the media staging of traditional, elite sports events.

Equally, developments in mobile technology allow amateur athletes to redefine their experience of sport via digital devices. The rise of ExerGamingi and Bio-Games (Millington 2012), which involve anything from downloading a mobile app to monitor one's daily runs, to GPS-enabled devices that allow users to gain insurance credits for physical activity, speaks to the shifts in how physical activity is being altered through new media. It also has shown how new media can create new physically engaged sub-cultures, as might be said of the Dance Dance Revolution style games that are found within arcades (Andrews 2006, Smith 2004). In these cases, the category of sport stretched via new media raising questions about the future interests of and populations within those communities.

One possible future involves the inclusion of computer games sports within mega-event programmes like the Olympic Games. After all, the synthesis of digital technologies and physical bodies - evident in technology trends - coupled with the emerging synergy between sports and new media makes this a scenario worthy of consideration. With the introduction of relatively rudimentary technologies like the Nintendo Wii and its derivatives, playing computer games is now very close to the physical and skill demands of many Olympic sports. In a boxing simulation game, players must throw their arms in similar ways to boxers in a ring. At the opposite end of the scale, in some games the simulator is the primary environment for learning the real activity, not just a substitute. Pilots may train for weeks within game simulators. Thus, the world of simulation is bringing the amateur and elite athlete closer and, when those worlds become indistinguishable, some aspects of the physical experience may be brought into question. Closely allied is the way that all aspects of the elite athlete's body today are monetized and digitized within simulation games. Computer games sports titles are among the most lucrative, not least because every season brings a new opportunity to 
re-sell a product. For instance, if one looks at FIFA Football games, it is apparent the players' biographies and histories within the game resemble actual players and the sponsorship present within the Game is also tied into wider relationships that exist around the presenting sports federation.

A number of challenges arise from these transformations, which threaten changes to the sports business. First, there are questions about equity and fairness, which often do not generate much public controversy, unlike unfair advantages that athletes may derive from, say, doping. Yet, athletes with access to the latest simulator or most sophisticated form of data capture may increase their chances of doing better in sport. For instance, consider the recently launched Google Glass, a thin device that would allow skiers, for instance, to wear goggles and have a built-in display into the glasses helping them anticipate turns, via GPS-enabled mapping built into the display. Would this kind of technology compromise the skills required for the sport, or might we see its use enrich the competitive experience in a way that technology has so many times before? In some respects, the acceptance of such use is part of the range of knowledge that is accepted as an enriching part of an athlete's development. In the same vein, one may argue that having a better coach, nutritionist or masseuse may also provide advantages that are unfair, insofar as not every athlete will have them.

Second, the increased use of ExerGaming or the development of serious games that effectively gamify physical activity may be incentives for people to take part in sports or other forms of activity, but may also become subject to wider public expectations. For instance, it is already apparent that some private insurance schemes and even gymnasia offer incentives for activity (Finkelstein \& Kosa 20). One might imagine further that digital surveillance of physical activity could become an even more appealing route through which to minimize risks taken by insurance firms, perhaps even a compulsory part of a policy. Companies might even require clients to wear health monitors to ensure they do all they can to avoid health risks. In such a world, one might have serious concerns about solidarity and individual liberty. Yet, there may also be a strong public health argument for providing such incentives.

The ability of new media to generate new communities of athletes is also a challenge for the sports world. While it is reasonably common to see sports with long histories being 
pushed out of mega-event programmes like the Olympic Games for newer sports such as BMX biking, these are no small matters for either sports federations or athletes. An athlete might have spent an entire career training in one sport and lose their means of competing on the basis of such a decision. Yet, the growth in computer game sports and the creation of new demographics of sports participation may force organizations and sponsors to reconsider what the landscape of sports participation should look like. Whereas today's sports funding remains dominated by male athletes in many countries, sports nurtured within digital worlds may create different kinds of competitions that are better at stimulating equal participation. They may even enable men and women to compete alongside each other more easily. Should this be possible, there would be a compelling argument for why funding should be directed to sports federations that prioritize these kinds of disciplines over others, in order to preserve other important values.

Finally, new media presents challenges over the means by which sponsorship is secured and generated around athletes and teams. On the approach to the London 2012 Olympic Games, this issue became particularly controversial around the freedom of athletes to mention non-Olympic products and brands within their social media profiles, which the IOC forbade in the interest of core financial sponsors of the Games. Rogers (2012) reports how athletes were using Twitter to protest against the IOC's rule, which specified athletes would not be allowed to undertake actions that were associated with commercial organizations. By using the Twitter hashtags \#Rule40 and \#wedemandchange, athletes expressed their concerns about not being able to promote their individual sponsor relations within personal social media platforms. Athletes may even take into their own hands their financial futures by utilizing social media in publicity and funding campaigns. For example, in 2012, the USOC track athlete Nick Symmonds built a campaign around auctioning space on his arm through eBay, where the winner would have a Twitter account name temporarily tattooed during the Olympic period. The example speaks to how sports may need to adapt to the new media world. More individual athletes may act as their own brands, rely less on a club's negotiation of sponsors, and generally have a greater capacity to act as their own mediators of financial support. Social media and digital technology generally provides much greater agency and opportunities to develop a brand with limited resources, which is 
unconnected to a product and, by implication, unconnected to a broader arrangement made on behalf of the athlete's team or club.

While the sports authorities appeal to the need to maintain exclusive and restrictive relationships with sponsors to deliver evens and, subsequently, is required to protect those interests by also restricting athletes freedom to pursue other commercial interests, there are questions about how far an athlete can be asked to jeopardize their own livelihood by neglecting their ongoing obligations and relationships. At the heart of this debate is a question about whether these new media environments are extension of commercial media space, or whether they are more like personal communication spaces. Twitter has (generally) no editorial structure that affects the things people say. In this sense, it is not directly analogous to a professional broadcaster, even though it is a commercial platform. Yet, it is also naïve for users to conclude that their content has no political currency, or that it can only be seen as personal expression. One complicating factor in resolving this matter is that content online now migrates from one place to another. Where previously an advertisement or form of communication existed within one space - a billboard, a radio station, a television channel - today content can be embedded into multiple locations, and this may make it difficult to ensure that no breaches of contract occur, if an athlete publishes something.

\section{THE SPECTATOR}

The second category of new media innovation concerns the affect on the spectator's experience. In 1995, Hemphill imagined the possibility of 'head cams' worn by people to locate themselves within the world and how these might be utilized as part of the sport experience. Twenty years later, the previously large apparatus of a head camera was mistaken in one crucial aspect - size. Today's prototypes that would make possible such scenarios are focused much more on the nanoscale and altering sensory experiences by adapting our biology, rather than appending an object to ourselves. Thus, there already exists prototype technology for digitally augmented glasses, using Google's Glass, which could permit digital content being shown through the device, transforming the eye into a digital interface. Recent developments in augmented reality indicate how the physical world will be layered with additional digital content within our field of vision. So, as spectators watch an ice hockey match wearing the lenses, they would be able to add layers of content, from data about the athletes, zoom in close to see their faces, or be 
able to monitor their physical state to know if, for instance, an athlete is fatigued. Where once such possibilities were the stuff of science fiction, today they are an imminent and, in some respects, realized aspect of sports competition.

In the interim, the population of spectators is expanding as a result of digital technology, which has increased the communal space in which shared sports spectatorship takes place - perhaps no more so than the erection of giant urban screens, which become live sites during important sports events. Yet, the small screen has also become a way of creating new audiences and new spectator experiences. Industry professionals now look to leveraging the 'second screen' (Cruickshank et al. 2007) within their sports broadcasting, especially as more people begin to consume sport live to mobile (REF). Indeed, far from taking viewers from television, consumption of content via the second screen has been shown to increase the television viewing hours of sports (Edgar, 2012). Equally, experiments within broadcasting are changing the conditions of sport spectatorship. Television broadcasters provide a comprehensive feed to all content, rather than just select the most iconic moments to broadcast, while viewers choose their own event programme.

Sports producers are also changing the landscape of the playing field through new media. Digital Replacement Billboards (Sprogis et al. 2001) is one example of how elements within the real world are transformed into screens within screens, allowing broadcasters to tailor what advertisements a television viewer sees. So, sports fans watching in South Korea would see different billboards within the field of play compared to someone in the United Kingdom. In this world, the physical space of the sports arena becomes literally a digital void, a blank unit populated with content only in the case of mediation.

The role of the spectator is also being altered by the rise of new media - social media in particular. Today's spectator is now a co-producer of sports news and entertainment, as status updates, tweets, video diaries, and photographs produced by spectators at events become part of the digital assets of an event. In this respect, their function also intersects with that of the professional media producers - journalists - creating a need for the media industries to both manage and marshal such content or risk jeopardizing their ability to leverage interest from their media privileges at sports events. 
The implications of digital technology reconfiguring the sports arena are enormous, raising challenges for how sports are produced and managed. Consider an example from the art world, which sheds light on how experiences within sports stadia may change. In 2010, the Museum of Modern Art in New York was occupied by two rebel artists who decided to stage their own exhibition within the gallery using augmented reality. Their intervention was titled 'We AR in MoMA,' but was not commissioned by or approved by the MoMA as one of its exhibitions. Instead, their art work was overlaid onto 'MoMA's "real” galleries using augmented reality technology' (Thiel, 2010), creating a rogue exhibition within their exhibition. Their actions provoked discussion about who owns physical space when our experience of it is mediated via a digital device, which is a crucial predicament for sports, where space is currency. In the case of the MoMA, visitors would walk around the gallery, activate their AR application on their digital devices, and witness floating objects alongside the exhibits. The implications of this are immense and present questions over who owns the digital signals within a building. Presently, there are no signal scramblers within sports events, but it is conceivable that digital innovation could allow companies to ambush the space within the arena. This is increasingly important as more of the physical experience is enriched via a mobile device. Spectators at sports may spend a considerable amount of their time looking at the playing field through a device - camera or phone - and so even the stadium is not an unmediated space.

The second screen in sports spectating also changes the kinds of organizations that are able to co-produce content. For example, in platforms like Twitter, the use of a hashtag (essentially a key word that becomes a collective way of capturing a conversation) can be a way for audiences to organize their communal experience and curate the news content it generates. This changes the media industry hierarchy considerably, compelling sports producers to adapt to these trends or face growing redundancy. In part, this is why, at the London 2012 Paralympic Games, spectators within the arena were invited to use Twitter hashtags to share content, with some of the tweets displayed on large screens within the venue in real-time. It is also why, as of this writing, large sports federations and organizations like the IOC are building relationships with digital innovators, which provide video sharing opportunities (e.g. YouTube), broad socialized media experiences (e.g. Facebook), microblogging platforms (e.g. Twitter), and 
photograph-sharing opportunities (e.g., Flickr and Instagram), so as to constantly ensure a presence within the most popular sharing platforms of the day (Miah \& Jones, 2010).

One may argue that these new media players are not disrupting the dominance of long term media broadcasters, but there is evidence of how much value can be accrued by using new media to access new markets. For instance, since Beijing 2008, the IOC has established relationships with Google to livestream sports content on YouTube within territories that have no rights agreement, principally Asia and Africa. Among the places included for both Beijing 2008 and London 2012 are Afghanistan, Bangladesh, Cambodia, and the Democratic Republic of Congo. Nevertheless, the expanding costs of winning the rights may also make it challenging for public broadcasters to compete with commercial broadcasters, which may result in limiting the amount of live sports audiences see. This has particular implications for host nations, as events like the Olympic Games involve considerable public cost. As such, to locate the televisual content behind a pay wall could jeopardize public support. This concern also reminds us of the importance of establishing compatible legal agreements around the sharing and broadcasting of content that underpins the possibility of change resulting from digital innovation. Indeed, many of the issues that limit the use of new media within sports hinge on matters of intellectual property. Whether it is sharing permissions at a sports event in digital form or challenges over territories, an understanding of international media law within these discussions is paramount.

Finally, regarding the increased use of social media by spectators, one of the problems was made apparent by the London 2012 Olympic Games Opening Ceremony where, the day after, British Member of Parliament Aiden Burley tweeted that the content was 'multicultural crap,' which caused a media storm. Burley defended his right to express a personal view, but neglectsed the fact that followers give value to his comment in part because he is an MP. In this sense, the example exemplifies the user's own naivety about whether any kind of public voice can be simply personal. The reactions to his comment indicate that it cannot, even if the Twitter biography includes a caveat indicating as much. Thus, the key point here is that social media collapses the remaining space of personal expression into a pervasive public sphere. In short - and to refer to early debates about the internet in academic literature - tweeting is not like going down the pub and having a chat, it is more like getting up on a soap box and making an 
argument. Yet, because social media occupies a place in our lives that is often outside of working hours or space, this distinction becomes blurred for users.

Another example of the challenges arising from social media is what happened after the initial diving performance of Team GB athlete Tom Daley. Very soon after, an abusive tweet from a spectator created controversy (Miah, 2012). Specifically, a 17 year old directed a message toward Daley's account saying 'you let that your dad down i hope you know that'. Daley's father passed away the previous year and was a central part of his career development and, while this may be considered by some to be simply bad taste, it led to a police inquiry and the arrest of the teenager. However, the twist in the tale is that the notoriety and fuss surrounding the message came not from the tweet itself, but by the fact that Daley included the message and the person's username within a re-tweet which read 'After giving it my all...you get idiot's [sic] sending me this...' Had Daley just ignored the message, it is unlikely that anyone would have noticed.

Even the mainstream media cannot avoid controversy related to social media, which changes the power relationships among media providers. One good example of this during the London 2012 Olympics was also the night of London 2012's Olympic Games opening ceremony, during which the hashtag \#NBCfail began to trend on Twitter due to criticisms over its coverage, a narrative that continued throughout the Olympic and Paralympic Games. NBC's perceived failing in deciding not to screen the ceremony live and cutting elements from their coverage led one British journalist to tweet the email address of the NBC Olympics President. He was subsequently suspended from Twitter (temporarily) for breaching their guidelines, which provoked a debate about whether this person's email was private. Yet, the more intriguing point is that these matters were played out entirely within a social media environment and a trending hashtag within Twitter can be an influential force within the news agenda. This example reveals how new media can expose gaps within the media system that allow citizens - either in their personal or public roles - to stage campaigns that call into question the operational practices of the media.

These examples speak to the challenges arising when people make public their comments about sport, which must be seen as part of a broader debate about how people negotiate their public and private lives within online environments. Yet, it also 
reminds us how athletes are becoming part of the spectator's entourage and the media coverage, as their comments on each others' performances becomes part of how the sports event is communicated and experienced. On numerous occasions during the London 2012 Games, direct quotes from commentators were lifted directly from their social media feeds, bypassing the interviewer process altogether.

\section{THE OFFICIALS}

A third dimension of sport that is changing through digital technology is the role of the officials. One change in this area is the introduction of artificial officiating devices, more often than not some kind of camera-based or laser technology to monitor the playing field with a view to making more accurate judgments over what is taking place. An early utilization of such technology was the Hawk-Eye surveillance system first used by the US Tennis Open in 2004. As Repanich (2010) describes,

The system works by mounting 10 high-speed cameras around the court with five dedicated to each side of the net to capture the ball's movement from multiple angles, measuring its speed and trajectory. Then a computer processes that information, pinpointing the spot on the court within $3 \mathrm{~mm}$ of where the ball hit the ground and calculating the ball's compression to determine the size and shape of the mark that represents where the ball touched the court.

One of the most significant changes in this regard - and one of the more longstanding debates - is the inclusion of goal line technology within football. Over the years, there has been resistance to using such technology for two principal reasons. The first is a concern over interrupting the flow of the game, while the second is on the financial cost. Yet, goal line technology is a relatively simple system compared to the number of other ways that digital innovation could increasingly regulate what happens within sports competitions. Even the use of microphones for athletes during competition demonstrates how relatively primitive digital technology can change the conditions of a playing field, for athletes and spectators.

Yet, technological advances are no guarantee of a better sport experience or even a more accurate measurement of what took place. For example, one of the controversies of the London 2012 Olympic Games was an incident in fencing whereby a failure for a countdown clock to function effectively meant that one competitor proceeded to a gold 
medal match, while another did not. In this case, it was a combination of human and technological error that led to the mistake. The example highlights a problem around digitalization within sports that is broader than the debate about deciding whether a human is more effective at officiating than a technological device. Rather, most technology of this kind implies a human-computer interaction, and the synergy of this relationship is crucial in ensuring that the right kinds of interests are promoted. It cannot be assumed that better digital technology implies better results. Furthermore, it cannot be assumed that automation removes the role of human judgment, since there is often a human somewhere in the loop interpreting what the technology says.

Many sports continue to resist the implementation of digital officials and other forms of innovation that may change the experience of a sport. In some cases, there may be legitimate ethical reasons why an athlete would seek to ensure that microphones are not required during competition. Sports are, after all, arenas of cultural endeavor where certain expectations of behavior that would otherwise be required of people are absent. Athletes can interact physically with others in a way that is not permissible in wider society. By implication, one might argue that the conduct of athletes within the playing field in terms of what they say should also be free from conditioning via digital surveillance. Yet, to the extent that athletes enjoy a life that is partly predicated on their being role models - on and off the field - then this view seems a relatively weak basis on which to maintain the sports field as a different kind of social space. Indeed, when athletes do transgress rules dramatically, wider civil or criminal laws may become a means of punishment, even if they took place within the playing field - one thinks of racial abuse, physical assault as primary examples.

Furthermore, the digital solution to an official's limited capacity need not be solved by digital innovation. For instance, football has also considered adding additional officials whose role is specifically to watch the goal line to see whether a ball has crossed or not. Thus, there may be human solutions to sport's problems that are less rooted in digital solutions. Yet, the digital imperative may often obscure other such solutions, perhaps in part due to its being a more economically desirable solution.

Besides camera and laser technology, smart phones may also prove to be another innovation that changes the way officials work. For instance, in 2012, the Mexican Indoor 
Football League utilized a new form of carding system, whereby the referee used a device to display red and yellow cards, rather than actual cards. This mobile app called RefereePro had the additional functionality of replacing pencil and paper as a way of tracking fouls and other statistics (Laird 2012).

\section{CONCLUSION}

As sports become further constituted by digital technologies and the user culture that surrounds them, their values and the experience change as well. These changes are presenting challenging implications for how sport is organized, commercialized and experienced. For instance, there may be a strong social argument to relocate some sports within solely virtual arenas, rather than physical worlds. The travel required of sports competitions is incompatible with the depleting resources in the world and the carbon emissions deriving from such travel. Sports that are dependent on natural resources, like golf, may not be possible without some kind of technological solution. At the same time, the high stakes of sport demands that technology improves the reliability of decisions that affect the outcome - who wins. In large part, this is why line surveillance technology have become such important aspects of the officiating process. In these cases, it is the inability of humans to ascertain what is happening in the field of play that leads humanity towards a digital era.

This chapter has considered ways in which new media in a very broad sense presents challenges for the production and practice of sports. It demarcated three territories as distinct categories - athletes, spectators and officials. Within these three categories, I have also considered aspects of the sports industries, such as sponsors and broadcasters and how their work may be challenged or enriched by some of the new processes within the media.

What made the London 2012 Games worthy of being called the first social media Olympics was the way that the sports reporting was driven by social media content. From the latest athlete's tweet, to Twitter audience polls on breakfast television coverage, social media became pervasive not just in terms of the volume of people using it, but how content within social media drove the news cycle When considering directions of new media within sport, recent years indicate a capacity within professional 
media to adapt to and appropriate new media environments and platforms, coupled with a migration to other spaces of consumption. Yet, control over the content remains a challenging issue for sports administrators and there remains resistance to completely opening channels of communication. This is most apparent within Twitter accounts where corporations may consider share someone else's content as an endorsement and where there is still a culture of following only those who are considered part of the sports family.

Nevertheless, perhaps the single most important issue in this aspect of sport's digital future is how the increased management of social communication will take place. For instance, if Tom Daley had been advised by his manager to ignore the abusive tweet, rather than re-tweet it himself, there might not have been the same kind of public outcry and distraction from his competition. Yet, a situation where all public communication requires approval in advance may be a world that is more impoverished and devoid of meaningful human interactions, both good and bad. This is also why the rules that govern public communication do not map neatly on to social media. It is the playing out of one's ideas in public and the capacity to redefine these without catastrophic judgment that allows a society to progress and flourish, rather than the excessive stifling of thoughts, even if they are ill-informed and misguided. 


\section{Bibliography}

Anderson, C., Gentile, D. and Buckley, K. (2007) Violent Video Game Effects on Children and Adolescents: Theory, Research, and Public Policy. Oxford: Oxford University Press.

Andrews, G. (2006) Land of a Couple of Dances: Global and Local Influences on Freestyle Play in Dance Dance Revolution, Fiber Culture, 8. Available Online: http://eight.fibreculture.org/fcj-048-land-of-a-couple-of-dances-global-and-localinfluences-on-freestyle-play-in-dance-dance-revolution Accessed: 5 November, 2012.

Bideau, B., Multon, F., Kulpa, R., Fradet, L. \& Arnaldi, B. (2004) Virtual reality applied to sports: do handball goalkeepers react realistically to simulated synthetic opponents?, VRCAl '04 Proceedings of the 2004 ACM SIGGRAPH international conference on Virtual Reality continuum and its applications in industry, pp. 210-216.

Byron, T. (2008) Safer Children in a Digital World: The Report of the Byron Review. Nottingham, DCSF Publications.

Comstock, G. and Scharrer, E. (2007) Media and the American Child. Academic Press.

Fairweather, B. (2002) Disembodied Sport: Ethical Issues of Virtual Sport, Eletronic Games, and Virtual Leisure. Research in Philosophy and Technology, 21.

Cruickshank, L., Tsekleves, E., Whitham, R., Hill, A. \& Kondo, K. (2007) Making Interactive TV Easier to Use: Interface Design for a Second Screen Approach. The Design Journal, 10(3), 41-53.

Edgar, A. (2012) Conference at Oxford.

Finkelstein, E.A. \& Kosa, K.M. (2003) Use of incentives to motivate healthy behaviors among employees. Gender /ssues, 21(3), 50-59.

Hemphill, D.A. (1995) Revisioning Sport Spectatorism. Journal of the Philosophy of Sport, XXII, 48-60. 
Huffman, R.K. \& Hubbard, M. (1996) A Motion based virtual reality training simulator for bobsled drivers. In S. Haake, ed. The Engineering of Sport. Netherlands: Balkema Publishing, 195-203.

Laird, S. (2012, Nov 15) Smartphone Replaces Red Cards in Pro Soccer Match, Mashable.com Available Online: http://mashable.com/2012/11/15/smartphone-soccerreferee Accessed: 10 March, 2013.

Levy, R.M. \& Katz, L. 2008) Virtual Reality Simulation: Bobsled and Luge, IACSS International Symposium Computer Science in Sport, 2007, available online: http://people.ucalgary.ca/ rmlevy/Publications/Levy_Katz_IACSS2007.pdf [Accessed: 15 October, 2012].

Marshall, S. J., Biddle, S. J. H., Gorely, T., Cameron, N. \& Murdy, I. (2004) Relationships between media use, body fatness and physical activity in children and youth: a metaanalysis. International Journal of Obesity, 28, 1238-1246.

Miah, A. (2012, August 10) London 2012: A social media Olympics to remember. BBC Online, Available: http://www.bbc.co.uk/news/technology-19191785 Miah, A. \& Jones, J. (2012) The Olympic Movement's New Media Revolution: Monetization, Open Media \& Intellectual Property. In Wagg, S. \& Lenskyj, H., Eds. Handbook of Olympic Studies, Palgrave, 274-288.

Mitchell, W. (1995) City of Bits : space, place, and the Infobahn. Cambridge Mass.: MIT Press.

Ott, M. \& Pozzi, F. (2011) Digital Games as Creativity Enablers for Children. Behaviour and Information Technlogy, 31(10), 1011-1019.

Repanich, J (2010) Can Cameras and Software Replace Referees? Popular Mechanics, http://www.popularmechanics.com/outdoors/sports/technology/cameras-fouls-andreferees Available Online. Last Accessed 1 April, 2013.

Rogers, K. (2012, July 31) Olympic athletes take to Twitter to rally against strict sponsorship rules. The Guardian. Available online: 
http://www.guardian.co.uk/sport/2012/jul/31/olympic-athletes-twitter-sponsorship-rules [Accessed: 5 April, 2013].

Smith, Jacob. (2004). I Can See Tomorrow In Your Dance: A study of Dance Dance Revolution and music video games. Journal of Popular Music Studies 16(1), 58-84.

Sprogis, P., Wilf, I., Tamir, M., and Sharir, A. (1996) Electronic Billboard Replacement Switching System. United States Patent No, US 6,191,825 B1, Date of Patent: Feb 20, 2001.

Theil, T. (2010) "ARt Critics Face Matrix, Available Online: http://www.missionbase.com/tamiko/We-AR-in-MoMA/index.html Last Accessed: 31 October, 2012. Walls, J., Bertrand, L., Gate, T. \& Saunders, N. (1998) Assessment of upwind dinghy sailing performance using a virtual reality dinghy sailing simulator. Journal of Science and Medicine in Sport, 1(2), 61-72. 


\section{NOTES}

i The term exergaming applies to any digital game experience that involves strenuous gross

motor activity, often within the sports genre. Exergames may be played within computer game

arcades, home consoles, or may be more like a pervasive game, where the experience is defined

by navigating a real physical world, using a mobile navigation device, as for geocaching. 